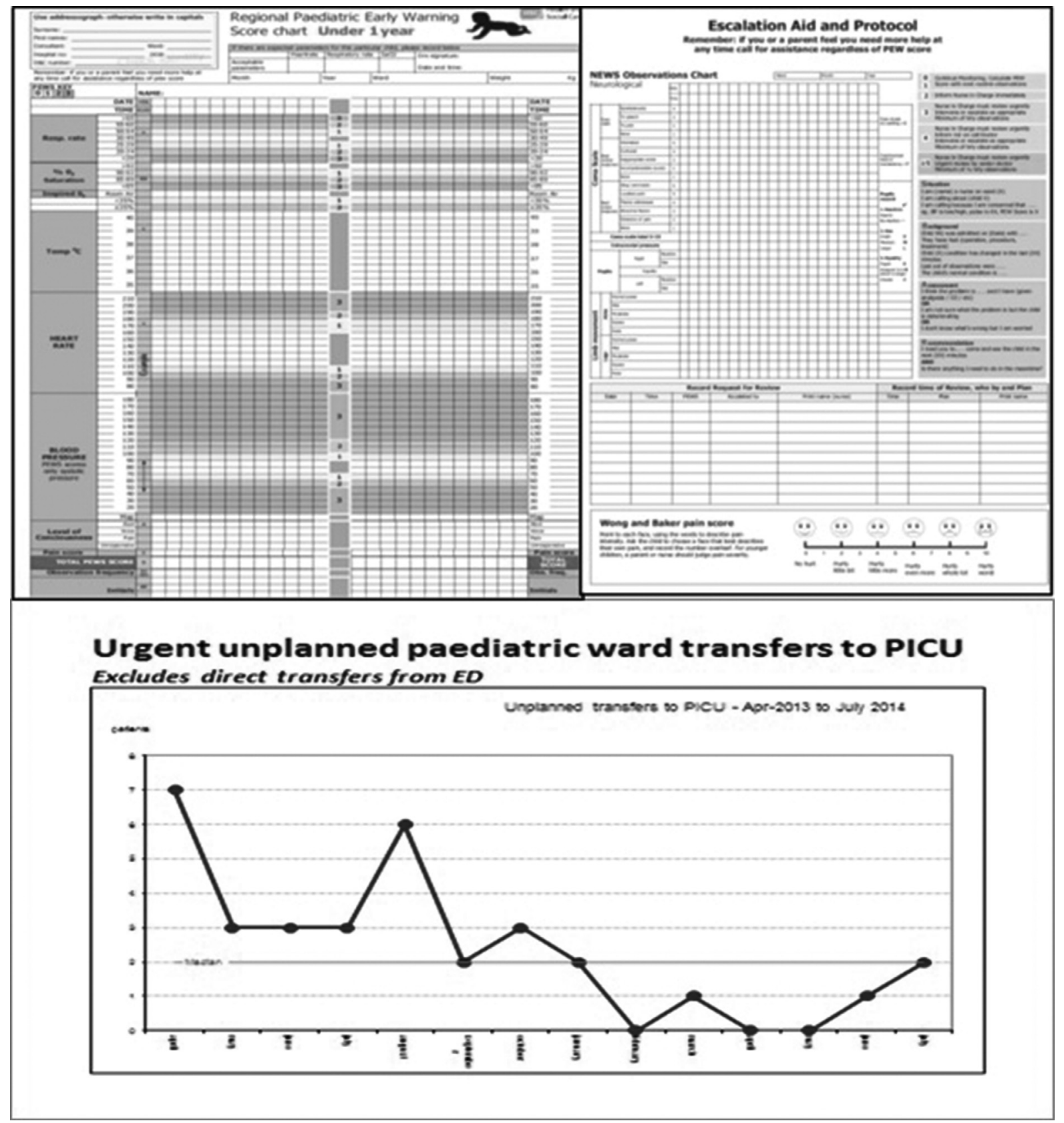

Abstract G296(P) Figure 2 Example of improvement work and early detection deterioration

multidisciplinary clinical team to the learning sessions and reported progress in an "all teach, all learn" environment. The Model for Improvement was used to test and implement changes.

Results Figure 1 Regional Driver Diagram and participating units

Figure 2 - Examples of Improvement work undertaken as part of the collaborative

Early detection of deterioration

Figure 3 - parent safety poster

Conclusion The value of a regional paediatric quality improvement collaborative is that it brings clinicians, managers and parents together with a set of core aims. The collaborative gets results by engaging frontline teams in identifying the key challenges faced, generating the potential solutions and then by using the methodology, testing these in real time to see what works and importantly what doesn't work before scaling up change.

\section{G297(P) USE OF EMAIL ADVICE - LINE TO IMPROVE MANAGEMENT OF CHILDREN IN THE COMMUNITY}

S Montgomery-Taylor, R Cotton, N Manek, M Watson, R Klaber. Paediatrics, Imperial College Healthcare NHS Trust, London, UK

10.1136/archdischild-2015-308599.274

Aims Our innovative integrated child - health programme puts the GP practice at its heart bringing specialist expertise and support into primary care. "Open access" is one key component and includes an email advice - line for community health - professionals to contact paediatricians for advice. Registrars wrote replies and the aim was to respond within 1 working day. The aim of this project was to evaluate the use and perceived effectiveness of the email advice line on management of children in the community. 


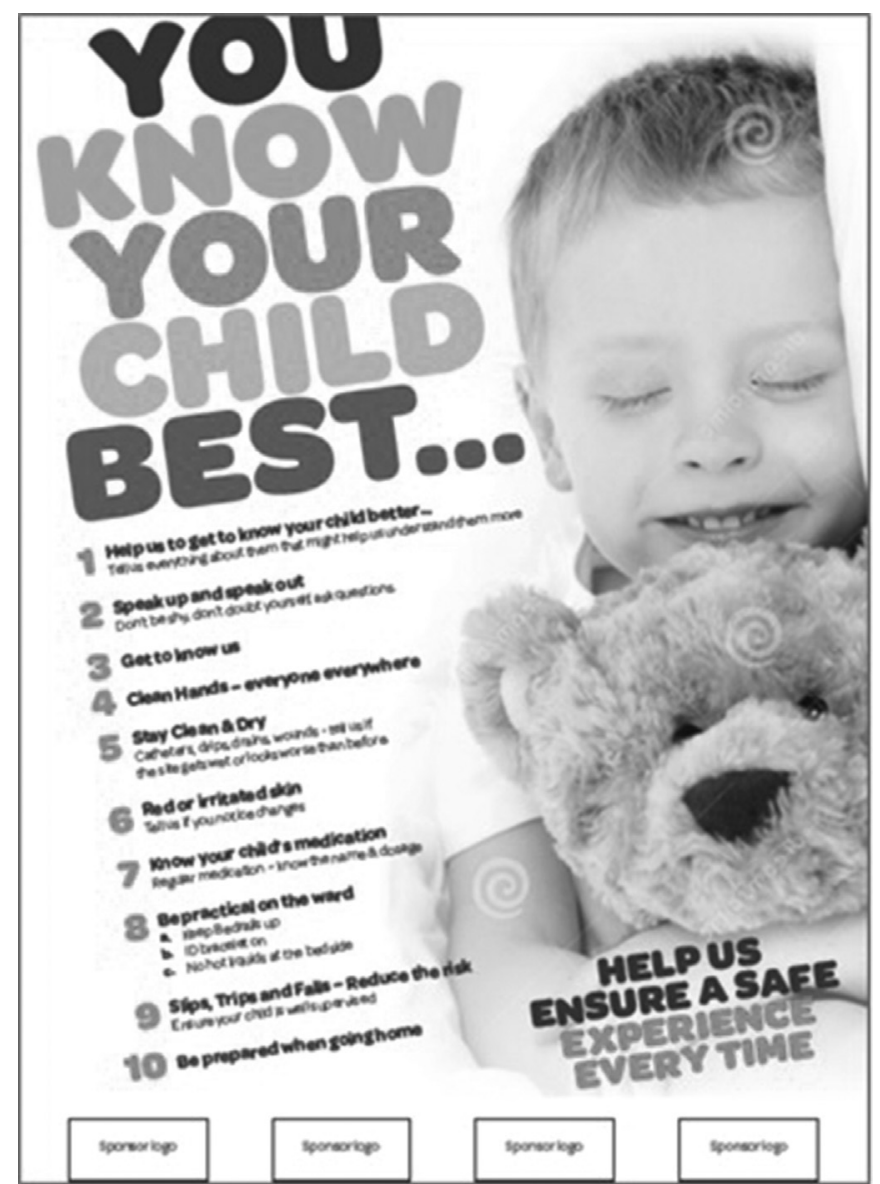

Abstract G296(P) Figure 3 Parent safety poster

Methods 451 emails sent by GPs and community health professionals over 10 months were reviewed. The final outcome of the email advice was categorised by three reviewers independently, with a paediatric consultant providing input when a consensus was not reached. Forty randomly selected community professionals and six paediatric registrars were contacted to provide feedback.

Results $99.6 \%$ of responses came from 91 GP practices in London. $62.7 \%$ of emails received a response within 1 working day. $55.2 \%$ of responses provided specialist paediatric advice to support the GP to continue managing the patient in primary care. $31 \%$ advised the GP to refer the patient to paediatric outpatients, while $7.1 \%$ were deemed to divert a potentially 'unnecessary referral' (Figure 1).

$81.8 \%$ of community health professionals agreed that the email advice line has reduced their frequency of hospital referrals. $100 \%$ agreed that they were satisfied with the responses received. Benefits of increasing knowledge in paediatric problems were also highlighted.

Six paediatric registrars felt that managing the responsibility of answering the emails alongside their clinical duties was feasible. 5/6 surveyed registrars also felt the advice line enhanced their own knowledge and learning.

Conclusion The email advice line supports the management of paediatric patients in the community and potentially reduces referrals. It enables services to be signposted and directs referrals more effectively, so that patients see the 'right person, right place, first time.' GPs valued the ease of access and efficiency of response for specialist advice, and patient

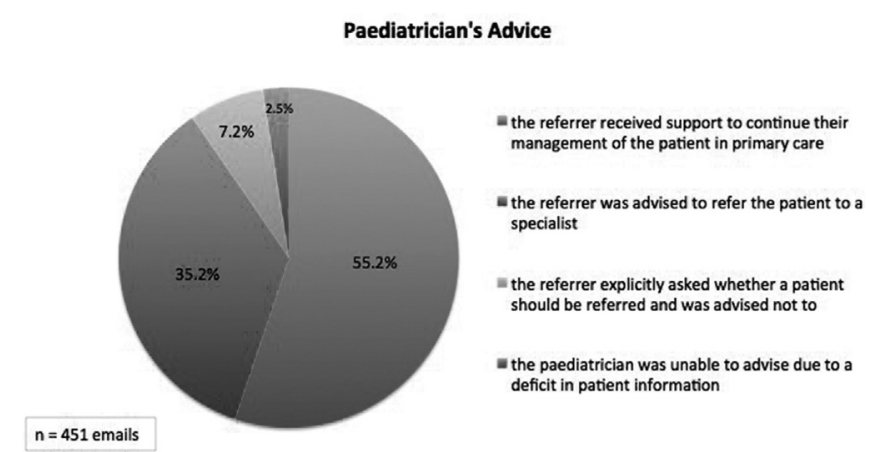

Abstract G297(P) Figure 1 Paediatrician's advice

satisfaction was reported by GPs as high. We believe this service empowers and enables GPs to manage paediatric patients with more confidence, and offers a unique opportunity to enhance education.

\section{G298(P) SACRAL DIMPLES IN THE NEWBORN - DOING LESS (AND SAVING MONEY) SAFELY}

${ }^{1}$ A Beh, ${ }^{1}$ A Rowland, ${ }^{1}$ P Reynolds, ${ }^{2} \mathrm{C}$ Bennett. ${ }^{1}$ Neonatal Department, St Peter's Hospital, Chertsey, UK; ${ }^{2}$ Radiology Department, St Peter's Hospital, Chertsey, UK

\subsection{6/archdischild-2015-308599.275}

Background Although spinal dysraphism is often detected antenatally, identifying certain signs postnatally, which might indicate increased possibility of an abnormality is important. Sacral dimples are common, but they are probably over-investigated. We set out to audit 4 years of ultrasound scans to see if investigations and follow up arrangements could be improved.

Methods All paediatric spinal ultrasound scans between Febuary 2009 - August 2013 were checked for origin, indication and result. Any with uncertain or positive scans also had MRI scan results and notes review.

Results There were 56 scans performed, aged 0 days to 22 months. The majority were referred from hospital doctors (75\%). Other sources included the orthopaedic clinic (16\%) and the patient's GP (9\%). Only 3 patients were found to have spinal dysraphism confirmed on US scan then MRI. From the 43 patients referred for investigation for a sacral dimple, none had spinal dysraphism. The three patients with spinal dysraphism all had either a skin tag or a hairy patch as an indication for the further investigation. From these results, and in keeping with available evidence, we have simplified the guideline for further investigation.

Clinical indicators used to refer for ultrasound scan - Figure 1

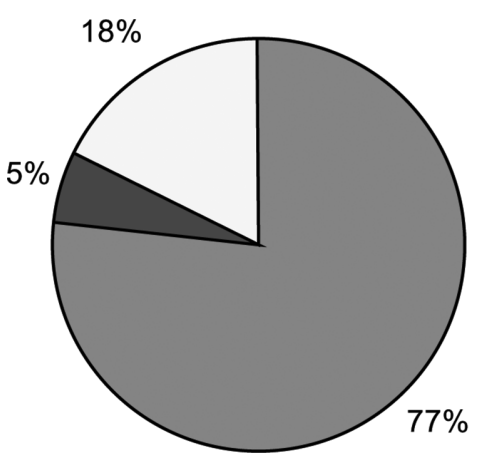

Sacral Dimple Skin tag $\square$ Other

Abstract G298(P) Figure 1 Clinical indicators used to refer for ultrasound scan 